\title{
Matrix metalloproteinase-9 in pediatric rheumatic heart disease with and without heart failure
}

\author{
AHMED A. ELHEWALA ${ }^{1}$, MOHAMMED SANAD ${ }^{1}$, ALSHIMAA M. SOLIMAN ${ }^{1}$, \\ MAY M. SAMI ${ }^{2}$ and ALSHYMAAA. AHMED ${ }^{2}$ \\ Departments of ${ }^{1}$ Pediatrics, ${ }^{2}$ Clinical Pathology, Faculty of Medicine, Zagazig \\ University, Zagazig, Al Sharqia Governorate 44519, Egypt
}

Received July 7, 2020; Accepted September 30, 2020

DOI: $10.3892 /$ br.2020.1380

\begin{abstract}
In cardiovascular disorders, the myocardium may be subjected to the breakdown and remodeling of collagen by metalloproteinase-9 (MMP-9). We hypothesized that the serum MMP-9 concentration may be elevated in pediatric patients with rheumatic heart disease (RHD) and heart failure (HF), and its level can be correlated with the HF severity. Thus, in the present study, we aimed to evaluate the sensitivity and accuracy of MMP-9 to predict HF in children with RHD and to determine its effectiveness as an indicator of the degree of HF. This study included 98 consecutive children admitted to the Department of Pediatrics, Zagazig University Hospital, Al Sharqia Governorate, Egypt with newly diagnosed RHD. Their ages ranged from 8.5 to 16 years. Fifty-eight children had RHD without HF while 40 children were complicated with HF which was diagnosed clinically and by echocardiography. A total of 44 healthy children were enrolled as a control group. MMP-9 serum levels were estimated by enzyme-linked immunosorbent assay. The serum MMP-9 concentration was higher in the RHD without HF and RHD with HF groups than this level noted in the control $(\mathrm{P}<0.001)$. MMP-9 was a significant predictor of $\mathrm{HF}$; area under the curve $(\mathrm{AUC})=0.85[95 \%$ confidence interval (CI), 0.76-0.94]. At the level of $386.9 \mathrm{ng} / \mathrm{ml}$, MMP-9
\end{abstract}

Correspondence to: Dr Alshymaa A. Ahmed, Department of Clinical Pathology, Faculty of Medicine, Zagazig University, Zagazig, Al Sharqia Governorate 44519, Egypt

E-mail: alshymaa2110@gmail.com

Abbreviations: ACS, acute coronary syndrome; ASO, antistreptolysin O; AUC, area under the curve; CVDs, cardiovascular diseases; CBC, complete blood counts; CI, confidence interval; $\mathrm{CRP}, \mathrm{C}$ reactive protein; $\mathrm{ECHO}$, echocardiogram; $\mathrm{EF}$, ejection fraction; FS, fractional shortening; HF, heart failure; MMPs, matrix metalloproteinases; OR, odds ratio; NPV, negative predictive value; PPV, positive predictive value; ROC, receiver operating characteristic curve; RF, rheumatic fever; RHD, rheumatic heart disease; TLC, total leukocyte count; WHO, World Health Organization

Key words: ejection fraction, heart failure, matrix metalloproteinase 9, rheumatic heart disease detected HF with a sensitivity 95\% (95\% CI, 83.08-99.39), specificity $74.14 \%$ (95\% CI, 60.96-84.74), positive predictive value $71.70 \%$ (95\% CI, 61.96-79.75), negative predictive value 95.56\% (95\% CI, 84.67-98.82) and accuracy $82.65 \%$ (95\% CI, 73.69-89.56). In addition, MMP-9 showed a significant negative correlation with ejection fraction and fractional shortening ( $\mathrm{P}=0.01$ and $\mathrm{P}=0.02$, respectively). In conclusion; MMP-9 may be an independent sensitive marker with which to detect HF in children with RHD and it can predict the prognoses of these patients as it correlates with the severity of HF. Further studies considering MMP-9 in the detection of 'silent' RHD in school aged children and asymptomatic HF in children with known RHD especially in rural areas, are highly recommended.

\section{Introduction}

Heart failure (HF) is defined as an abnormal cardiac function and/or structure that lead to the inability of the heart to deliver oxygen to the tissues at a rate that compensates for their metabolic requirements. A major cause of pediatric HF in developing countries is rheumatic fever (RF) and rheumatic heart disease (RHD) (1). RF and RHD present serious problems worldwide according to the Global Burden of Diseases in 2015 (2). There have been crucial efforts by the World Health Organization (WHO) to prevent and control RHD (3). Improving the quality of housing and reducing overcrowding in combination with providing penicillin for early management of streptococcal infections are the most important steps needed to eradicate RHD (4). While there has been an evident decline in its prevalence in Egypt since the 1950s, acute rheumatic fever (ARF) and RHD remain national issues, and efforts by the Egyptian authorities to control ARF and RHD are ongoing (5).

Despite advancements in diagnostic work-up techniques, patients with RHD and HF may be diagnosed late or undiagnosed in developing countries due to the lack of echocardiography and experienced cardiologists in rural health centers. Therefore, sensitive and reliable serum markers as a simple laboratory test in conjunction with echocardiography are required for early detection of RHD and HF (6). The importance of studying cardiac biomarkers in the diagnosis and prognostic stratification of children with HF has been well emphasized; these biomarkers may act as future therapeutic 
targets (7). Similarly, noninvasive tools to monitor patients with HF before the occurrence of clinical deterioration are also required (8).

Matrix metalloproteinases (MMPs) are zinc-dependent endopeptidases that belong to the metzincin superfamily. MMPs have a key role in tissue remodeling in cardiovascular diseases (CVDs). MMPs are a promising target of therapy, as the administration of doxycycline 'a tetracycline antibiotic that acts as a nonspecific MMP inhibitor' in myocardial infarction patients improved ischemia/perfusion injury. Further studies on the implications of specific MMPs in different CVDs are required to clarify their role in disease progression and to aid the discovery of new therapeutic targets (9).

Gelatinases (MMP-2 and MMP-9) are the most frequently analyzed MMPs in HF. According to the majority of studies, active myocardium remodeling is associated with increased activity and concentrations of MMP-2 and MMP-9, which can be useful markers to identify patients at risk for HF development, and as indicators of patient outcome (10). In one study; circulating MMP-9, in contrast to MMP-2, was the only predictor of late-onset congestive HF (11).

We hypothesized that the serum MMP-9 concentration may be elevated in pediatric patients with RHD and HF, and its level can be correlated with the HF severity. Thus, the aim of the present study was to evaluate the sensitivity and accuracy of the MMP-9 level to predict HF development in children with RHD and to determine its effectiveness to indicate the degree of HF as detected by echocardiography.

\section{Patients and methods}

Patients. This prospective study was conducted at the Pediatric Cardiology Unit, Pediatrics Department, Zagazig University Hospital, Al Sharqia Governorate, Egypt, from September 2015 to September 2019. The patients eligible for inclusion were consecutive children with age $\leq 16$ years of either sex and from the same Egyptian ethnicity. The patients were newly diagnosed with RHD by clinical presentation and echocardiography based on the World Heart Federation criteria (12) and whose parents agreed to provide written informed consent for participation. Patients with underlying lung pathologies, bronchial asthma, congenital heart diseases (CHDs), cardiomyopathy, aortic aneurisms, end organ failure, cancers, or other complications of RHD such as atrial fibrillation, pulmonary hypertension and thromboembolic disorders were excluded from the study.

A total of 125 children with newly diagnosed RHD were enrolled in the study. However, 27 were excluded: 4 patients had CHDs; 7 had lung disease according to X-ray examinations; 9 had coexisting complications; 4 had increased serum creatinine levels; and 3 had inadequate serum samples. Ultimately, this study included 98 patients with a median age (range) of 13.5 (8.5-16) years. A total of 40 patients (43.2\%) had comorbid $\mathrm{HF}$ at presentation, while the remaining 58 (56.8\%) had RHD without HF. The control group included 44 healthy, age- and sex-matched children.

Patient groups. Patients were categorized into two groups: RHD without HF and RHD with HF. This categorization was based on the presence of clinical symptoms and signs of HF, including dyspnea, orthopnea, lower limb edema, ascites, and intolerance to exercise with left systolic dysfunction, which was defined as an ejection fraction $(\mathrm{EF}) \leq 55 \%$ as estimated by transthoracic echocardiography (13).

Data collection and clinical examination. All patients reported their full medical histories, including history of rheumatic fever, and underwent general and thorough cardiac examinations. Children with recurrent ARF were diagnosed according to the revised Jones criteria 2015 (14).

Ethical consideration. Ethical approval for this study was obtained from Zagazig University Institutional Research Board (ZU-IRB) (approval no. 6005-23-6-2019). Written informed consent was obtained from the parents of the children included in the study. All procedures were performed according to the Declarations of Helsinki (https://www.who. int/bulletin/archives/79(4)373.pdf).

Sample size calculation. The sample size was calculated by applying the Buderer N 1996 formula (15) at a 95\% confidence interval (CI), $95 \%$ power, $98 \%$ sensitivity and an expected prevalence of $34 \%$ (16). An additional $10 \%$ was added to the calculated sample size to compensate for dropout.

\section{Methods}

Laboratory investigation. Complete blood counts were determined with Sysmex KX-21 hematology analyzer (Sysmex Corp.). C reactive protein (CRP) and antistreptolysin O (ASO) levels were determined using automated nephelometry $\mathrm{BN}$ Prospec (Siemens, UK) and turbidimeteric assay (Cobas 6,000, Roche Diagnostics, Deutschland), respectively. The normal range for CRP is up to $5 \mathrm{mg} / \mathrm{dl}$, and that for ASO is up to $200 \mathrm{IU} / \mathrm{ml}$. Liver function tests and kidney function tests were performed using Cobas Integra 400 Plus (Roche Diagnostics). All tests were performed at the Hematology and Chemistry Units, Zagazig University Hospital Laboratories.

Measurement of MMP-9 serum levels. Plain tubes were used to collect blood samples from patients when they were admitted to the hospital with suspected RHD. Sera were separated and stored at $-80^{\circ} \mathrm{C}$ until analysis. MMP-9 was analyzed using a commercial MMP-9 ELISA kit (Sunred Biological Technology), which applies the double antibody sandwich ELISA technique (17) according to the manufacturer's guidelines. The laboratory staff was blinded to the clinical and echocardiography data of the patients.

\section{Imaging techniques}

Plain chest $X$-ray in the postro-anterior view. This imaging was performed to detect cardiomegaly and to exclude other chest diseases.

Echocardiography. Conventional detailed echocardiography was performed in the Cardiology Unit of the Department of Pediatrics, Zagazig University Hospital. Ventricular systolic function assessment, chamber dimensions, wall thickness, left atrial diameter, left ventricular end diastolic diameter, left ventricular end systolic diameter, fractional shortening (FS), and EF were all measured using M-mode echocardiography. 
Pulsed or continuous wave Doppler ultrasound was used to assess the pressure gradient across the stenotic or regurgitated flow through the valves as previously described (18).

Classification of patients with HF. Based on the EF, patients were classified as mild HF, EF 40-54\%; moderate HF, 30-39\%; or severe $\mathrm{HF}, \mathrm{EF}<30$. In addition, patients were classified by the application of the modified Ross classification (19).

Statistical analysis. The data were analyzed using SPSS version 20.0 (IBM Corp.). Shapiro-Wilk test was performed to detect the normality of the data. Parametric numeric data are expressed as means \pm standard deviation and non-parametric data are expressed as medians and ranges. The unpaired Student t-test and one way analysis of variance with Fisher's Least Significant Difference (LSD) test were used to compare means; the Mann-Whitney U and Kruskal-Wallis tests with Dunn-Bonferroni post hoc method were used to compare medians. Categorical variables were compared using the Chi square test and Fisher's exact test when frequencies were less than five. Univariate and multivariate regression analyses were performed using the binary logistic regression test to evaluate MMP-9 as an indicator of HF. The numerical variables were dichotomized according to their medians or means. A receiver operating characteristic (ROC) curve was set up to calculate the area under curve (AUC) at 95\% CI. The level of serum MMP-9 with the maximum accuracy was determined as the best cutoff value. Correlations between variables were tested using the Spearman correlation coefficient (r). Results were considered significant when $\mathrm{P}<0.05$.

\section{Results}

Both patient groups were homogenous regarding demographic features (Table I). A total of $59(60.0 \%)$ patients provided a past history (one to two years ago) of RF. The past history of $\mathrm{RF}$, clinical manifestations, and distribution of valve lesions among patients are represented in Table SI.

The mean serum MMP-9 levels showed highly significant differences between the healthy controls $(61.0 \pm 22.8 \mathrm{ng} / \mathrm{ml})$ and the children with RHD without HF $(356.5 \pm 74.7 \mathrm{ng} / \mathrm{ml})$, between the healthy controls and children with RHD and HF $(422.3 \pm 63.5 \mathrm{ng} / \mathrm{ml})$, and between children with RHD without $\mathrm{HF}$ and children with RHD and HF ( $\mathrm{P}<0.001$ for each) (Table SII).

The univariate analysis showed that the serum MMP-9 level was associated with the risk of HF development [odds ratio (OR), 3.2; 95\% CI, 1.3-7.3, P=0.008). Potential confounders from the univariate analysis (Table II) including hemoglobin ( $\mathrm{Hb}) \%$, TLC, and ASO were introduced in a multivariate model, and the multivariate analysis revealed that MMP-9 was an independent indicator of HF in children with RHD (OR, 2.6; 95\% CI, 1.0-6.7, $\mathrm{P}=0.04$ ) (Table II).

The AUC of MMP-9 in patients was 0.85 (95\% CI, 0.76-0.94) (Fig. 1). The MMP-9 level of $386.9 \mathrm{ng} / \mathrm{ml}$ was considered the best cutoff point with the maximal diagnostic accuracy to predict HF in children with RHD. At this level, MMP-9 predicted HF with 95\% (95\% CI, 83.08-99.39) sensitivity, $74.14 \%$ (95\% CI, 60.96-84.74) specificity, a positive predictive value of $71.70 \%$ (95\% CI, 61.96-79.75), a negative
Table I. Demographic characteristics of the patient groups.

\begin{tabular}{|c|c|c|c|}
\hline Variable & $\begin{array}{l}\text { RHD without HF } \\
{[n=58(56.8 \%)]}\end{array}$ & $\begin{array}{l}\text { RHD with HF } \\
{[n=40(43.2 \%)]}\end{array}$ & P-value \\
\hline Age (year) & & & 0.30 \\
\hline Median (range) & $13.5(8.5-16)$ & $13.5(10.5-15.5)$ & \\
\hline Body weight (kg) & & & 0.06 \\
\hline Median (range) & $32.5(21-49)$ & $35.0(23-51)$ & \\
\hline Body mass index & & & 0.30 \\
\hline Median (range) & $18.5(13.9-27.2)$ & $19.3(15.8-23.5)$ & \\
\hline Sex, n (\%) & & & 0.09 \\
\hline Male $(n=45)$ & $23(39.7)$ & $22(55.0)$ & \\
\hline Female $(n=53)$ & $35(60.3)$ & $18(45.0)$ & \\
\hline Residence, n (\%) & & & 0.50 \\
\hline Urban $(n=13)$ & 8 (13.7) & $5(12.5)$ & \\
\hline Rural (n=85) & $50(86.3)$ & $35(87.5)$ & \\
\hline
\end{tabular}

Mann-Whitney $\mathrm{U}$ test and Chi square test were used to compare medians and frequencies, respectively. RHD, rheumatic heart disease; $\mathrm{HF}$, heart failure.

predictive value of $95.56 \%$ (95\% CI, 84.67-98.82) and $82.65 \%$ (95\% CI, 73.69-89.56) accuracy. Cross tabulation of the patients is shown in Table SIII.

There was no significant correlation in any of the parameters, including the duration of symptoms, CRP and ASO titer (Table SIV). MMP-9 appeared to significantly increase with the deterioration of $\mathrm{EF}$ and $\mathrm{FS}(\mathrm{P}=0.01$ and $\mathrm{P}=0.02$, respectively) (Fig. 2; data not shown). In this cohort; 33 mild and 7 moderate cases were included. The mean \pm SD MMP-9 was $413 \pm 62$ and $464 \pm 53$, respectively $(\mathrm{P}=0.055)$. By the application of the modified Ross classification; 36 patients were in Class II and 4 patients were in Class III. The mean \pm SD MMP-9 was $416 \pm 63$ and $478 \pm 29$, respectively $(\mathrm{P}=0.059)$.

\section{Discussion}

In the present study, serum matrix metalloproteinase 9 (MMP-9) concentrations did not correlate with $\mathrm{C}$ reactive protein (CRP) levels in any group. Previous studies considering MMP-9 in rheumatic heart disease (RHD) that can support our results are rare. Previous research has shown that CRP has a positive feedback on serum MMP-9. For example, Cimmino et al examined the effect of CRP on MMP-9 gene expression in vitro in vascular smooth muscle and in vivo in patients with acute coronary syndrome (ACS) (20). Harfoosh et al detected a positive correlation between MMP-9 and CRP serum levels in patients with ACS (21). Although ACS encompasses myocardial inflammation and remodeling, the definite pathology could differ from that in RHD and HF. This can explain the contrast between our results and those of other studies.

In the present study, the mean serum MMP-9 level in the control group was lower than that in both the RHD without heart failure (HF) and RHD with HF groups, indicating that this serum marker can be a promising screening tool for 
Table II. Univariate and multivariate analyses of the predictors of heart failure.

\begin{tabular}{lccccccr}
\hline & \multicolumn{3}{c}{ Univariate analysis $^{\mathrm{b}}$} & & \multicolumn{3}{c}{ Multivariate analysis $^{\mathrm{c}}$} \\
\cline { 2 - 3 } Factors $^{\mathrm{a}}$ & OR & $95 \%$ CI & P-value & & OR & $95 \%$ CI & P-value \\
\hline $\mathrm{Hb} \geq 11.8$ & 0.3 & $0.13-0.73$ & $\mathbf{0 . 0 0 8}$ & & 0.3 & $0.1-0.9$ & $\mathbf{0 . 0 5}$ \\
$\mathrm{TLC} \geq 13.2$ & 3.2 & $1.4-7.5$ & $\mathbf{0 . 0 0 6}$ & & 3.3 & $1.3-8.6$ & $\mathbf{0 . 0 1}$ \\
$\mathrm{ASO} \geq 178.5$ & 3.1 & $1.3-7.3$ & $\mathbf{0 . 0 0 8}$ & & 3.1 & $1.2-7.9$ & $\mathbf{0 . 0 2}$ \\
$\mathrm{MMP}-9 \geq 383.3$ & 3.2 & $1.3-7.3$ & $\mathbf{0 . 0 0 8}$ & & 2.6 & $1.0-6.7$ & $\mathbf{0 . 0 4}$ \\
\hline
\end{tabular}

${ }^{\mathrm{a}}$ All numerical variables were dichotomized based on the median levels in patients except for MMP-9 which was dichotomized based on the mean value. ${ }^{b}$ Univariate analysis included all variables that showed significant differences among children with RHD without HF and children with RHD and HF. ${ }^{c}$ Multivariate analysis included all variable that were significant indicators in the univariate analysis. Significant P-values are shown in bold print. RHD, rheumatic heart diseases; HF, heart failure; Hb, hemoglobin; TLC, total leukocyte count; ASO, antistreptolysin O; MMP-9, matrix metalloproteinase 9.

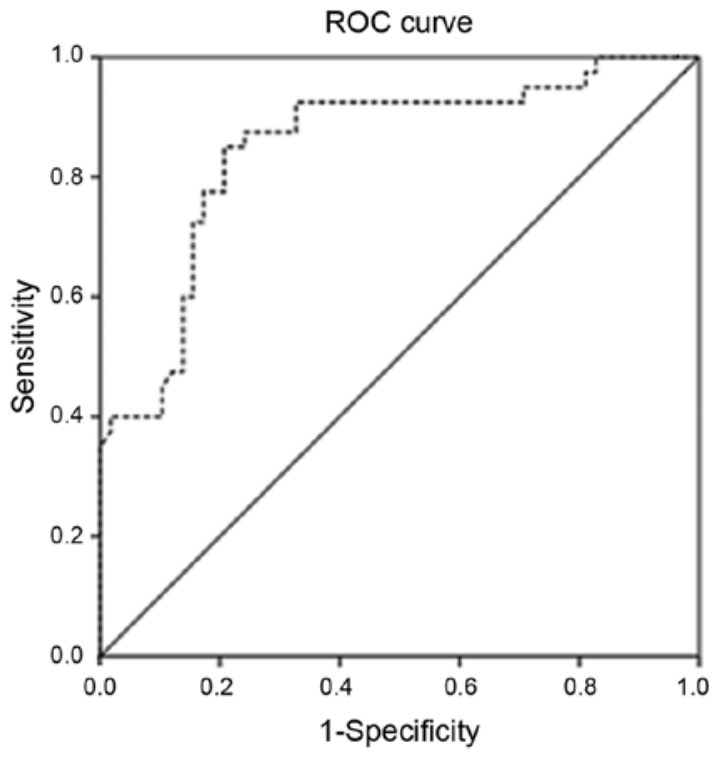

Figure 1. ROC curve for MMP-9 in the detection of HF in RHD. Serum MMP-9 concentration as a significant predictor of HF in children with RHD. Area under curve $=0.85(0.76-0.94)$ at $95 \%$ CI. ROC, receiver operating characteristic curve; MMP-9 matrix metalloproteinase-9; RHD, rheumatic heart disease; CI, confidence interval.

subclinical RHD among school-aged children particularly in high risk endemic areas in conjunction with echocardiography. Although echocardiography has superiority in detecting subclinical RHD, Dougherty et al stated that portable echocardiography is expensive, heavy to transport, with limited battery capacity and functionality; in addition, it requires well-trained cardiologists or medical staff members (16).

Serum MMP-9 at a cutoff point $386.9 \mathrm{ng} / \mathrm{ml}$ was found to be a sensitive predictor of HF in children with RHD. Zhao et al (22) found that MMP-9 is overexpressed in the myocardium of patients with RHD congestive HF. Most of the studies concerning MMP-9 in HF, such as studies by Abou-Raya et al (23) Collier et al (24) and Morishita et al (25), suggest that active myocardial remodeling is associated with increased MMP-9 concentrations (10).

Lee et al reported that serum MMP-9 concentrations were significantly higher in patients with RHD $>30$ years of

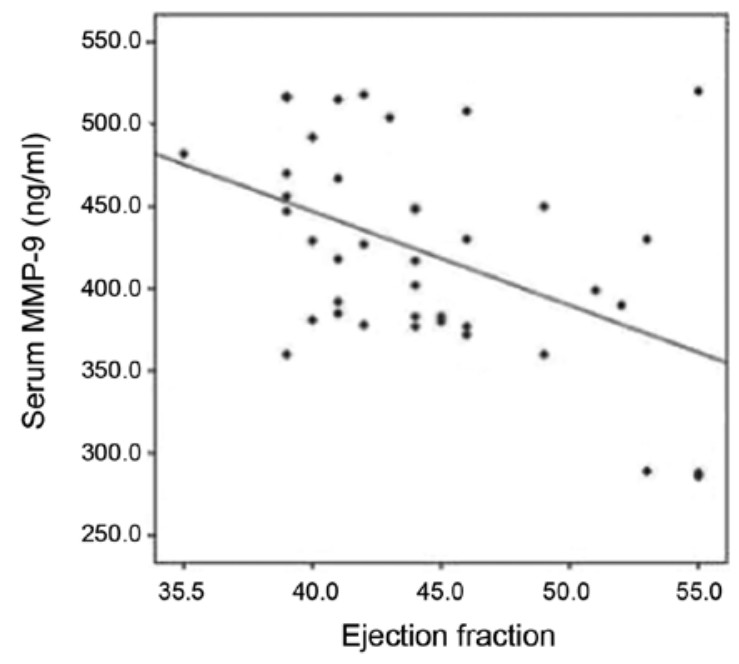

Figure 2. Scatter plot with line chart for the correlation between MMP-9 and EF. MMP-9 levels increased significantly with the deterioration of EF in the RHD with HF group. MMP-9 matrix metalloproteinase-9; EF ejection fraction; RHD, rheumatic heart disease; HF, heart failure.

age, when compared to the controls, and in a patient group of $<30$ years there was no significant difference. These results are contradictory against ours, and can be explained by the small sample size in that group which included 10 patients and 10 age-matched controls (26).

Kantor and Rusconi reported that HF in childhood and young adolescence often presents at an advanced stage; thus, biomarkers that can support traditional diagnostic tools are valuable. These authors mentioned MMPs as possible markers but the existing data were not sufficient (27).

Similarly, de Couto et al adopted the term 'pre-heart failure' as the process of cardiac remodeling indicated by the drop of ejection fraction (EF) which precedes the appearance of symptoms by years, gains from the early interruption and reversal of cardiac remodeling is greater than just treating symptoms (28). Therefore, the follow-up of children with RHD by MMP-9 can predict the decline in left ventricular systolic dysfunction early before symptoms enabling the early intervention before overt irreversible failure occurs. 
In the present study, serum levels of MMP-9 were negatively correlated with EF and fractional shortening (FS). These results were also demonstrated by Yan et al (29) who reported that elevated MMP-9 levels were connected with the deterioration in left ventricular (LF) function. According to Yoshihisa et al (30); reduced EF is an independent predictor of increased cardiac event rates. The authors stated that the initial assessment of LVEF is important for deciding treatment and predicting prognosis. Based on this; we assumed that the serum MMP-9 level may be associated with the prognostic status of patients. Radosinska et al (10) proposed that the MMP-9 level may not only be a useful marker to identify patients at risk for HF development but also, it can serve as an indicator of patient outcome. According to Kantor and Rusconi, the most challenging point in pediatric HF is the choice of treatments. This may be an additional value of biomarkers, which may guide decisions of how to manage and predict patient outcomes (27).

To the best of our knowledge; MMP-9 has not been previously studied in pediatric patients with RHD either in or outside Egypt, at the time of writing this manuscript. Therefore, this study adds to our general understading regarding the contribution of MMP-9 to the pathogenesis of RHD and HF in children and draws attention to a new marker and target of therapy for these patients.

On limitation of the present study was that it was conducted on symptomatic patients in a single center; the sample size was also relatively small. Thus, further multicenter studies with larger cohorts involving other types of MMPs are required to confirm these results. Future studies concerning MMP-9 in the screening of school-aged children for subclinical RHD in comparison with echocardiography, and screening of children with known RHD for asymptomatic cardiac dysfunction to allow early and effective intervention before the development of overt $\mathrm{HF}$ are highly recommended. Further investigations including the correlation of MMPs with other cardiac biomarkers such as natriuretic peptides (atrial or brain subtypes) or high sensitivity CRP, the severity of symptoms and valve lesions, prospective follow-up of children with HF to study the correlation between MMPs and the degree of deterioration in cardiac function to examine their role in monitoring these patients, and clinical trials to investigate the effects of doxycycline in children suffering from RHD and HF are also recommended.

In conclusion, the MMP-9 serum level can be considered as an independent sensitive indicator with which to identify children with RHD who are at risk of HF events. MMP-9 can be used to assess patients with HF; increased levels may indicate the need to intensify patient medical therapy. MMP-9 was higher in children with RHD either with or without HF than in healthy controls; therefore, MMP-9 is a promising diagnostic marker of subclinical RHD in school-aged children, although further studies are required.

\section{Acknowledgements}

Not applicable.

\section{Funding}

This research did not receive any specific grant from funding agencies in the public, commercial, or not-for-profit sectors. All the authors have indicated they have no financial relationships relevant to this article to disclose.

\section{Availability of data and materials}

Data are available upon reasonable request from the corresponding author.

\section{Authors' contributions}

The requirements for authorship have been met by all authors. All authors contributed to the study conception and design. The original idea was conceived by AAE and MS. AAE and MS also revised the manuscript before the publication. AMS contributed to the patient selection, history taking, data collection and blood sample withdrawal; under the supervision of AAE and MS. MMS contributed to the data analysis, manuscript writing, and plagiarism checking. AAA contributed to sample processing, results collection, data analysis, manuscript writing and submission for publication. All authors certify that personally wrote at least $90 \%$ of the manuscript. Finally, the manuscript was read and approved by all the authors. All authors are responsible for the reported research.

\section{Ethics approval and consent to participate}

Ethics approval for this study was obtained from Zagazig University Institutional Research Board (ZU-IRB) (approval no.6005-23-6-2019). All procedures were performed according to the Declaration of Helsinki. Signed informed consent was obtained from the parents of the involved children.

\section{Patient consent for publication}

Not applicable.

\section{Competing interests}

All the authors have no competing interest to declare.

\section{Authors' information}

Alshymaa A. Ahmed: ORCID ID: 0000-0002-8695-4037.

\section{References}

1. Chaturvedi V and Saxena A: Heart failure in children: Clinical aspect and management. Indian J Pediatr 76: 195-205, 2009.

2. Watkins DA, Johnson CO, Colquhoun SM, Karthikeyan G, Beaton A, Bukhman G, Forouzanfar MH, Longenecker CT, Mayosi BM, Mensah GA, et al: Global, regional, and national burden of rheumatic heart disease, 1990-2015. N Engl J Med 377: 713-722, 2017.

3. Dougherty S, Beaton A, Nascimento BR, Zühlke LJ, Khorsandi M and Wilson N: Prevention and control of rheumatic heart disease: Overcoming core challenges in resource-poor environments. Ann Pediatr Cardiol 11: 68-78, 2018.

4. Carapetis JR, Beaton A, Cunningham MW, Guilherme L, Karthikeyan G, Mayosi BM, Sable C, Steer A, Wilson N, Wyber R and Zühlke L: Acute rheumatic fever and rheumatic heart disease. Nat Rev Dis Primers 2: 15084, 2016.

5. Sorour KA: Rheumatic heart disease in Egypt: Gloomy past and promising future. Egypt Heart J 66: 139-142, 2014. 
6. Sarkar S, Rastogi M, Chaudhary P, Kumar R, Arora P, Sagar V, Sahni IS, Shethi S, Thakur K, Ailawadhi S, et al: Association of rheumatic fever \& rheumatic heart disease with plausible early \& late-stage disease markers. Indian J Med Res 145: 758-766, 2017.

7. Fernandes BA, Maher KO and Deshpande SR: Cardiac biomarkers in pediatric heart disease: A state of art review. World J Cardiol 8: 719-727, 2016.

8. Nadar SK and Shaikh MM: Biomarkers in routine heart failure clinical care. Card Fail Rev 5: 50-56, 2019.

9. Azevedo A, Prado AF, Antonio RC, Issa JP and Gerlach RF Matrix metalloproteinases are involved in cardiovascular diseases. Basic Clin Pharmacol Toxicol 115: 301-314, 2014.

10. Radosinska J, Barancik M and Vrbjar N: Heart failure and role of circulating MMP-2 and MMP-9. Panminerva Med 59: 241-253, 2017.

11. Wagner DR, Delagardelle C, Ernens I, Rouy D, Vaillant M and Beissel J: Matrix metalloproteinase-9 is a marker of heart failure after acute myocardial infarction. J Card Fail 12: 66-72, 2006.

12. Reményi B, Wilson N, Steer A, Ferreira B, Kado J, Kumar K, Lawrenson J, Maguire G, Marijon E, Mirabel M, et al: World heart federation criteria for echocardiographic diagnosis of rheumatic heart disease-an evidence-based guideline. Nat Rev Cardiol 9: 297-309, 2012.

13. Jayaprasad N: Heart failure in children. Heart views 17: 92-99, 2016.

14. Szczygielska I, Hernik E, Kołodziejczyk B, Gazda A, Maślińska $\mathrm{M}$ and Gietka P: Rheumatic fever-new diagnostic criteria. Reumatologia 56: 37-41, 2018.

15. Juneja A and Sharma S: Issues of sample size in sensitivity and specificity analysis with special reference to oncology. J Cancer Res Ther 11: 482-484, 2015.

16. Dougherty S, Khorsandi M and Herbst P: Rheumatic heart disease screening: Current concepts and challenges. Ann Pediatr Cardiol 10: 39-49, 2017.

17. Isik A, Gursul C, Peker K, Aydın M, Firat D and Yılmaz I: Metalloproteinases and their inhibitors in patients with inguinal hernia. World J Surg 41: 1259-1266, 2017.

18. Koestenberger M, Friedberg MK, Nestaas E, Michel-Behnke I and Hansmann G: Transthoracic echocardiography in the evaluation of pediatric pulmonary hypertension and ventricular dysfunction. Pulm Circ 6: 15-29, 2016.

19. Ross RD: The ross classification for heart failure in children after 25 years: A review and an age-stratified revision. Pediatr Cardiol 33: 1295-1300, 2012.

20. Cimmino G, Ragni M, Cirillo P, Petrillo G, Loffredo F, Chiariello M, Gresele P, Falcinelli E and Golino P: C-reactive protein induces expression of matrix metalloproteinase-9: A possible link between inflammation and plaque rupture. Int J Cardiol 168: 981-986, 2013.
21. Harfoosh AK, Elaziz AFA, Mahmoud E and Saleh A: Matrix metalloproteinase-9 as a potential biomarker for inflammatory events in acute coronary syndrome, and its relation to diabetes mellitus in these patients. Al-Azhar Assiut Med J 13 (Suppl 1) S71-S80, 2015.

22. Zhao Y,Zhou X, Liao X and Yang Z: Expression and significance of matrix metalloproteinase-1,9, tissue inhibitor of metalloproteinase-4 and extracellular matrix metalloproteinase inducer in the myocardium of congestive heart failure in patients with rheumatic heart diseases. Zhong Nan Da Xue Xue Bao Yi Xue Ban 34: 790-795, 2009 (In Chinese)

23. Abou-Raya S, Naim A and Marzouk S: Cardiac matrix remodelling in congestive heart failure: The role of matrix metalloproteinases. Clin Invest Med 27: 93-100, 2004.

24. Collier P, Watson CJ, Voon V, Phelan D, Jan A, Mak G, Martos R, Baugh JA, Ledwidge MT and McDonald KM: Can emerging biomarkers of myocardial remodelling identify asymptomatic hypertensive patients at risk for diastolic dysfunction and diastolic heart failure? Eur J Heart Fail 13: 1087-1095, 2011.

25. Morishita T, Uzui H, Mitsuke Y, Amaya N, Kaseno K, Ishida K, Fukuoka Y, Ikeda H, Tama N and Yamazaki T: Association between matrix metalloproteinase- 9 and worsening heart failure events in patients with chronic heart failure. ESC Heart Fail 4: 321-330, 2017.

26. Lee SD, Chen LM, Kuo WW, Shu WT, Kuo WH, Huang EJ, Tsai CC, Li PC, Liu JY, Chen TH and Huang CY: Serum insulin-like growth factor-axis and matrix metalloproteinases in patients with rheumatic arthritis or rheumatic heart disease. Clin Chim Acta 367: 62-68, 2006.

27. Kantor PF and Rusconi P: Biomarkers in pediatric heart failure: Their role in diagnosis and evaluating disease progression. Prog Pediatr Cardiol 31: 53-57, 2011.

28. de Couto G, Ouzounian M and Liu PP: Early detection of myocardial dysfunction and heart failure. Nat Rev Cardiol 7: 334-44, 2010

29. Yan AT, Yan RT, Spinale FG, Afzal R, Gunasinghe HR, Arnold M, Demers C, McKelvie RS and Liu PP: Plasma matrix metalloproteinase-9 level is correlated with left ventricular volumes and ejection fraction in patients with heart failure. J Card Fail 12: 514-519, 2006.

30. Yoshihisa A, Sato Y, Kanno Y, Takiguchi M, Yokokawa T, Abe S, Misaka T, Sato T, Oikawa M, Kobayashi A, et al: Prognostic impacts of changes in left ventricular ejection fraction in heart failure patients with preserved left ventricular ejection fraction. Open Heart 7: e001112, 2020. 